\title{
El discurso de la bioconstrucción arquitectónica: divulgación y legitimación en revistas profesionales
}

The discourse of architectural bioconstruction: popularization and legitimation in professional journals

ANNA I. MONTESINOS LÓPEZ

UNIVERSITAT POLITÈCNICA DE VALÈNCIA

Recibido el 23/01/2014

Aceptado el 15/04/2014

ABSTRACT: This study analyzes various professional journals form the field of architecture and construction in order to examine how the discourse of the bioconstruction is introduced and legitimatized. In this textual genre, the construction of the professionalization of this emerging discipline is carried out through various discursive strategies such as didacticity, metaphorization and ideological legitimization.

Keywords: professional journals, specialised discourse, bio-construction, didacticity, metaphor, ideology.

RESUMEN: En este trabajo se analizan diversas revistas profesionales del campo de la arquitectura y la construcción con el fin de estudiar de qué manera se introduce y se legitima el discurso de la bioconstrucción. El proceso de la profesionalización de esta disciplina emergente se lleva a cabo mediante diversas estrategias discursivas como la didacticidad, la metaforización y la legitimación ideológica.

Palabras clave: revistas profesionales, discurso especializado, bioconstrucción, didacticidad, metáfora, ideología.

\section{Introducción}

Hoy por hoy, el interés por el medio ambiente ha llegado a todos los ámbitos y áreas del saber y, precisamente, una de las disciplinas más contaminantes es la construcción, por lo tanto, no podía quedar fuera de los ámbitos de máxi- 
ma preocupación. Así, por ejemplo, está emergiendo una incipiente filosofía de diseño de viviendas que utiliza alta tecnología y lo que se podría denominar alta naturaleza que se basa en el ahorro energético, el uso de materiales inocuos para el medio ambiente y la aplicación de los principios del diseño biofílico a fin de fomentar la salud, la energía y la belleza (Louv, 2013: 214). No es baladí que el año 2012 fuese el año de la bioconstrucción y que el 2013 fuese el de la eficiencia energética.

Así pues, en el ámbito de la arquitectura, la arquitectura técnica y la ingeniería de la edificación hay actualmente un proceso de profesionalización por lo que se refiere a la bioconstrucción y a la bioarquitectura, las cuales están haciéndose un espacio propio. Este proceso implica una cierta competencia con otras profesiones a la vez que un esfuerzo por institucionalizarse dentro de determinadas disciplinas.

El discurso de la bioconstrucción es hoy en día un discurso en proceso de elaboración incluso dentro de los ámbitos más especializados y profesionales. La cultura profesional de los arquitectos técnicos, aparejadores e ingenieros de la edificación está experimentando una transformación que va pareja con las transformaciones económicas y sociales, puesto que se está produciendo un cambio necesario en los materiales de construcción, las tecnologías y las técnicas constructivas, con el fin de adaptarse a las nuevas demandas sociales, principalmente debidas a la preocupación, cada vez mayor, por el medio ambiente. De aquí que ahora la consigna intertextual sea «pensar globalmente, construir y plantar localmente» (Louv, 2013: 174). Es precisamente este hecho el que da lugar a nuevas subdisciplinas. Las transformaciones sociales demandan nuevas formas de institucionalización y propician nuevas especialidades desde el conocimiento, en un proceso de reformulación de las categorizaciones profesionales previas.

Siguiendo la propuesta de Salvador, Macián y Marín (2013: 79), las prácticas discursivas constituyen un medio privilegiado de construcción de las profesiones y por ello su estudio nos provee de un instrumental precioso para la conceptualización y explicación de la sociología de las mismas.

En este trabajo se pretende analizar el género textual de las revistas profesionales de la construcción y de la arquitectura, con el objetivo de establecer la manera con que se introducen las especialidades emergentes de la bioconstrucción y de la bioarquitectura a través del discurso entre los profesionales. 


\section{Las revistas profesionales de la bioconstrucción}

Las revistas profesionales son un género textual donde se combinan los propósitos de investigación estricta con los de información, divulgación y contacto entre profesionales. Las revistas técnicas profesionales constituyen el canal de comunicación de la comunidad discursiva profesional, en las cuales se activan los mecanismos de información y de participación entre los integrantes de la comunidad, donde se fija una terminología propia y donde se desarrolla el discurso profesional. Así, el género se revela como una perfecta herramienta de análisis de las situaciones sociales en las que se produce la comunicación especializada (García Izquierdo, 2007: 122).

Veamos a continuación un fragmento de un apartado que lleva por título «Una nueva oportunidad profesional»:

Los técnicos y prescriptores del sector de la construcción tienen hoy el reto de adaptarse a las nuevas exigencias consecuencia de una legislación cada vez más exigente en el campo del medio ambiente y con una opinión pública cada día más sensibilizada.

Al igual que sucede en otros ámbitos de especialización del sector de la construcción, este tema va a comportar, según los actores de la industria, la aparición de un nuevo perfil de profesional experto en «ambientalización de la construcción que actuará como soporte al proyectista». Xavier Casanovas lo llama el «dietista del edificio», un profesional preparado para mejorar la calidad y las prestaciones de un edificio, interpretando y utilizando la amplia y compleja información que ofrecen las DAP. (7)

Aquí podemos ver algunos aspectos discursivos que desarrollaremos más adelante, como la metaforización (el «dietista del edificio») y la terminología técnica (DAP). Lo que queremos destacar es cómo se introduce la concienciación sobre el medio ambiente entre los profesionales y la generación de una nueva especialidad profesional.

En primer lugar, existe el reto de adaptarse a las nuevas exigencias de la legislación medioambiental; en segundo lugar, hay una opinión pública más sensible hacia el cuidado del medio ambiente; en tercer lugar, la sensibilidad y la legislación sobre la conservación del medio ambiente van a ser positivas para la profesión porque nace un nuevo perfil y supone, como se dice en el título del apartado, «una nueva oportunidad profesional»; en cuarto lugar, el nuevo profesional nace para mejorar la construcción; finalmente, este profesional lo conseguirá mediante la aplicación de las declaraciones ambientales de producto. Así pues, la disciplina genera más conocimiento, y no se trata de un conocimiento cualquiera sino que es un saber que, por un lado, al aplicarse mejora la opinión 
pública y, por otro lado, da la oportunidad de positivar la autoimagen grupal y profesional, a la vez que se plantean nuevas vías de profesionalización.

Estas revistas profesionales constituyen el macrogénero discursivo (García, 2007: 121; Salvador et al. 2013: 86) donde se establecen los criterios éticos, discursivos, profesionales, etc., se comparten los conocimientos relativos a la profesión de la construcción y de la arquitectura, y se reconocen los patrones textuales como propios por parte de la comunidad discursiva implicada (Montalt, 2005: 72). De esta manera, se utilizan fórmulas y referencias hacia el mismo colectivo profesional como se puede ver a continuación:

Dentro del ámbito que afecta a nuestra profesión (la edificación en todas sus vertientes), la bioconstrucción responde perfectamente a las finalidades a implantar, dado que desarrolla la filosofía óptima de convivencia entre los usuarios de los edificios, el medio ambiente que los rodea y la planificación y crecimiento sostenible de nuestras ciudades. (1)

En este caso, «Dentro del ámbito que afecta a nuestra profesión» centra el discurso medioambiental en la edificación con el claro objetivo de informar y sobre todo convencer al lector de las bondades de la bioconstrucción.

Así pues, hemos estudiado nueve revistas de la profesión de la construcción y de la arquitectura, como producciones discursivas regulares con reconocimiento entre los expertos, caracterizadas con propósitos investigadores, divulgadores, informadores y de contacto entre profesionales ya que son estos expertos los que constituyen el punto de referencia para los individuos que utilicen esta variedad, podemos hablar, en este sentido, de autolegitimación del enunciador (Montesinos, 2002: 83).

Podemos establecer la siguiente clasificación de las revistas analizadas según su procedencia y su tratamiento total o parcial de la bioconstrucción. En primer lugar, están las revistas de los colegios profesionales (BIA, La Punxa, $T A G)$; en segundo lugar, las revistas técnicas profesionales de la arquitectura y la construcción (Informes de la construcción, Inmueble, Cemento y hormigón, Arte y cemento); y, finalmente, las revistas profesionales dedicadas exclusivamente a la bioconstrucción (EcoHabitar, Ecoconstrucción). Desde el punto de vista de su frecuencia, la mayoría son bimestrales o trimestrales, aunque también las hay mensuales como EcoHabitar y, incluso, quincenales como Arte y cemento.

En general, en las revistas técnicas de los profesionales se encuentran algunos artículos relacionados con las especialidades emergentes como son, en nuestro caso, la bioconstrucción y la bioarquitectura. En este tipo de revista, el artículo aparece generalmente en un apartado específico y dedicado al tra- 
tamiento especial que tiene por tratarse de un tema novedoso y puntero. Este apartado se suele llamar Construcción sostenible, Arquitectura sostenible y Sostenibilidad, según las publicaciones analizadas. La bioconstrucción también se puede encontrar junto con otros artículos formando un monográfico que tiene diferentes aspectos importantes, como sucede en la revista Ecoconstrucción, cuyo contenido está destinado a los profesionales de la construcción, el urbanismo, la rehabilitación y el diseño sostenibles, lo que incluye arquitectos, aparejadores, ingenieros, consultores, fabricantes, instaladores, constructores promotores empresas de servicios energéticos, administraciones públicas, asociaciones del sector y empresas e individuos que busquen soluciones sostenibles y energéticamente eficientes. En este caso, el género textual tiene una estructura estable siempre dedicado al tema de la sostenibilidad y la bioconstrucción. Esta estructura es la siguiente: en primer lugar un apartado dedicado a lo más novedoso llamado Actualidad; en segundo lugar, el apartado dedicado a Ferias, que son el centro de encuentro de los profesionales y de presentación de las novedades de las empresas; en tercer lugar, suele haber un apartado dedicado a la Entrevista a un profesional destacado dentro de la disciplina; en cuarto lugar, encontramos el grueso de la revista compuesto por los artículos, organizados en temas monográficos. Así, por ejemplo, el número 32 de esta revista se divide en tres partes: Ahorro y eficiencia energética en edificación, materiales sostenibles y arquitectura sostenible; cada una contiene 8, 6 y 2 artículos, respectivamente. Finalmente, hay que destacar un apartado, considerado en el sumario, llamado Anunciantes, donde se puede ver la relación de anuncios de empresas que hay a lo largo de la revista.

En este caso y cuando se recoge en un mismo número los parámetros más importantes de un tema tan nuevo dentro de la profesión del constructor o del arquitecto, podemos decir que la bioconstrucción ha logrado una identidad autónoma con un canal de expresión exclusivo para esta disciplina, puesto que anteriormente o en la mayoría de los casos no se ha tratado más que de manera transversal y comprobamos que en otras publicaciones es simplemente un apartado, como ocurre en las revistas de los colegios de arquitectos, arquitectos técnicos, ingenieros de edificación y aparejadores.

Así pues, se pretende incidir de una manera profunda en el tema de la bioconstrucción. En cambio, en otras revistas profesionales donde el tema no está tan afianzado, se pretende también concienciar al lector sobre la importancia y la transcendencia de las disciplinas emergentes, mientras que en las revistas profesionales monográficas se supone un convencimiento y concienciación hacia el medio ambiente y, por lo tanto, se incide menos en esto. En otras palabras, hay artículos donde exclusivamente se busca convencer al lector para que 
cambie de opinión y se conciencie sobre el tema y, en otros casos, se describen las técnicas o los materiales utilizados en este tipo de construcción, no tanto para concienciar sino para informar una vez ya se ha implicado al lector. Aunque también es cierto que a menudo a las nuevas técnicas constructivas o a los biomateriales de construcción se les acompaña de algunas ideas que conviene tener en cuenta sobre estos, puesto que existe la creencia entre los profesionales de que los materiales sostenibles durarán menos o que resultará mucho más caro un proyecto bioconstructivo. Hablaríamos en ese caso de textos mixtos, donde se busca aceptar el cambio y las posibles soluciones para las nuevas situaciones sostenibles.

En el artículo «Nuevo planteamiento constructivo. Eficiencia y sostenibilidad» se concluye que para implantar los conceptos biosostenibles hace falta el cambio de la cultura de consumo de nuestra sociedad, además de la conciencia medioambiental en la construcción, que se hace expansiva más allá de nuestra vivienda, haciendo del mundo nuestra casa:

Si no empezamos a cambiar nuestros hábitos relacionados no solo con el uso de nuestra vivienda y con nuestro edificio, sino también con el entorno que nos rodea, será tremendamente difícil implantar o mejorar la eficiencia de los mismos.

Debemos empezar a cuidar y pensar en que nuestra vivienda no se circunscribe a las paredes exteriores, sino que nuestro edificio, urbanización, barrio, calle, parque y, en definitiva, todo el entorno que nos rodea, forma parte de nuestra casa, y debemos cuidarla y respetarla como tal. Si no empezamos a cuidar esto, pronto empezaremos a ver un fin cada vez más cercano. Sin afán de ser apocalíptico, sí es necesario recuperar esta olvidada conciencia social. Y cuanto antes, mejor. La actual situación nos brinda una oportunidad que no debemos ni podemos perder. Necesitamos un futuro racional y sostenible. Precisamente, por eso, el Colegio de Aparejadores de Madrid, volcado en la formación y la excelencia de sus técnicos, cuenta con un espacio y una iniciativa específica para fomentar estos criterios: 2012 El año de la Bioconstrucción. (1)

El discurso avanza hacia el reclamo urgente de una conciencia que lleve a recuperar un entorno constructivo más eficiente y sostenible, hecho que se convierte en una necesidad: "Necesitamos un futuro racional y sostenible». Al final del fragmento y del artículo se presenta una solución a lo que se ha expuesto, mediante un colectivo profesional y su labor renovada. 


\section{La didacticidad del discurso}

La didacticidad es de amplio interés en el estudio que aqui se presenta. El concepto de didacticidad pretende ampliar el de la mera didáctica, que se restringiría a discursos determinados por instituciones escolares o educativas como una parte de un currículum académico (Salvador et al., 2010: 253). Así, el concepto se extiende a otros ámbitos no académicos ni estrictamente didácticos donde se pretende hacer comprender conocimientos consolidados en una comunidad discursiva. En este sentido, y a pesar de que en las revistas profesionales se supone la existencia de un paradigma de corresponsabilidad entre los participantes, hay elementos de didacticidad, dado que se produce una transformación en el paradigma establecido hasta hace poco tiempo en las disciplinas de la arquitectura y de la construcción.

Se realizan algunas operaciones que llevan al profesional a hacer comprensible el nuevo paradigma a los otros profesionales, con el objetivo de integrar las nuevas nociones en la competencia de los lectores y en los saberes compartidos por la profesión. Por lo tanto, podemos decir que hay un proceso de profesionalización de la bioconstrucción, una transformación de la cultura profesional de los profesionales de la construcción y de la edificación.

Tratamos a continuación tres ejes donde observamos la didacticidad del discurso y mediante qué mecanismos se da a conocer la nueva disciplina. Estos son las definiciones, la terminología y los procesos metafóricos.

\subsection{Las definiciones}

Según Salvador (2009), la tarea comunicativa de definir debe ajustarse a una serie de convenciones que cada género establece. En nuestro estudio, el género revista profesional está dirigido a los especialistas, profesionales y colegiados de la arquitectura y de la construcción y, en principio, la dimensión didáctica es muy débil, puesto que el propósito es ofrecer una síntesis de los últimos avances de la investigación sobre el tema para la actualización de los conocimientos de los profesionales. En este sentido, en algunos casos se prevé que el lector conoce los términos específicos de las subdisciplinas bioarquitectura y bioconstrucción, ya que se habla de conceptos mediante siglas especializadas, mientras que en otros se presupone que el lector puede que no los conozca todavía y, por tanto, la sigla se presenta en el texto junto con su término desarrollado correspondiente. Veamos el ejemplo siguiente, extraído del apartado «Reportaje: Sostenibilidad» de la revista Arte y cemento (7, 2012: 
110-113), con el artículo «Declaraciones Ambientales de Productos: materiales que definen a un edificio sostenible»: «Las EPD (Enviroment Product Declarations) o DAP (Declaración Ambiental de Producto) son las herramientas que ofrecen esta valiosa información» (7).

A causa del contexto de especialización, nos sorprenden las reformulaciones de la sigla $E P D$, tanto por lo que se refiere al término completo correspondiente, en inglés, como por la traducción de la sigla al castellano y su correspondiente término desarrollado. Podríamos pensar que la publicación es antigua o bien que se trata de los inicios de este concepto y su término. Pero no se trata de nada de esto, sino que el autor conoce el contexto de la publicación, en este caso la revista profesional Arte y cemento, donde de forma extraordinaria se ha incluido un reportaje con información sobre sostenibilidad. Y los lectores, aunque profesionales, pueden desconocer los nuevos planteamientos sostenibles, ya que el cemento no se caracteriza, precisamente, por ser una buena solución medioambiental. Se produce un ajuste contextual y podemos hablar de pragmática léxica (Yus, 2007: 81, 86) de los lenguajes de especialidad, donde tanto el hablante como el oyente han de establecer hipótesis acerca de la accesibilidad de ambos a la información contextual necesaria para acceder a la interpretación pretendida.

Asimismo, la definición de cemento que ofrece una empresa que presenta su cemento menos contaminante sirve para iniciar un artículo, dejando claro que se sabe que la producción de este es poco sostenible, con la finalidad de argumentar que ha desarrollado otros cementos menos contaminantes. Veámoslo en el artículo «Proyecto Aether: nueva gama de cementos con bajas emisiones» de la revista Bioconstrucción:

El cemento, ingrediente clave del hormigón, es fundamental en la construcción, pero su producción genera emisiones $\mathrm{CO}_{2}$, al igual que sucede con otros materiales. Lafarge, uno de los líderes mundiales en materiales de construcción, ha desarrollado una nueva gama de cementos con bajas emisiones de carbono Aether. (9.e)

El caso contrario es una definición donde se busca la presentación de nueva información sin más, y se define al principio del texto, como se puede ver en el artículo «Bioconstrucción, vivienda sana y sostenible»:

La Domobiótica es una disciplina integral que integra la Arquitectura ecológica con los criterios biológicos de la Geobiología y la Bioconstrucción, y evita los excesos tecnológicos de la domótica en ciertos edificios inteligentes, esto exige unos requisitos biológicos mínimos en nuestro edificio. (4) 
Hasta ahora, hemos visto que la forma verbal principal para definir es la tercera persona del verbo ser, aunque no es extraño encontrar definiciones con la omisión del verbo, como vemos ahora en: «Tejados de madera, una solución tradicional para la bioconstrucción moderna» (2.b).

Otras veces, después de definir lo que es una disciplina y para concluir el texto se define lo que no es, como podemos ver en el ejemplo siguiente, donde la definición de lo que no es sirve para justificar que la disciplina no puede aplicarse cuando se quiere, sino cuando se puede, como se ve en el artículo «Geobiología y bioconstrucción, 1. a parte»:

Hemos de aclarar que la Geobiología no es una panacea llena de recetas que podemos aplicar en todo momento, o de cualquier manera, como muchas veces algunas personas pretenden, para adaptar lo que hay a sus propias necesidades. (2.c)

Para acabar, la definición se introduce también al final de los textos con el fín de reformular una idea expresada anteriormente, mediante una forma conclusiva, y con la intención de hacer comprender totalmente y de forma correcta al lector dicha idea. Veamos dos ejemplos de este tipo:

En definitiva, la bioconstrucción se podría definir como la ciencia que estudia las múltiples interacciones existentes entre el ser humano, su entorno residencial y laboral. No es una especialidad delimitada a una sola disciplina, sino más bien el conjunto de muchas relacionadas entre sí. Para la bioconstrucción es una prioridad la realización de un diseño artístico, saludable y sostenible mediante el que se pretende encontrar un equilibrio entre la cultura arquitectónica de un pueblo, sus técnicas de construcción y la investigación para el desarrollo de mejores soluciones constructivas con materiales autóctonos. La bioconstrucción, por tanto, no es una materia que esté estrictamente delimitada a una sola especialidad, pero no cumpliría con sus objetivos si no se contempla, se estudia y se enseña como una materia autónoma y especializada en todo su conjunto. (1)

La reformulación trata tanto de favorecer la difusión de la información como las posibles aclaraciones sobre esta. En el primer párrafo, se produce una reformación textual mediante la recapitulación (Bach, 2001: 254), donde el conector en definitiva vehicula una evidente recapitulación de la información sobre la bioconstrucción expuesta anteriormente. En el segundo párrafo, el conector por tanto, también tiene la función recapitulativa, pero lo hace por contraste respecto de lo que no es, de forma aclaratoria. 


\subsection{La difusión de la terminología}

Un buen porcentaje de la terminología de la bioconstrucción y la bioarquitectura está estrechamente relacionado con la terminología de la conservación del medio ambiente, que a su vez ha sido ampliamente popularizada los últimos años en todos los ámbitos divulgativos.

Así pues, como apunta Cabré (1999: 141), se trata de una concepción más abierta que asume la circularidad del saber y la circulación permanente de los términos especializados entre lenguaje común y ámbitos especializados, y de estos entre sí. La didacticidad, en este sentido, según Salvador (2002: 118) es una de las zonas privilegiadas de negociación semiótica, en el marco de la circulación de los saberes, mientras que las unidades terminológicas son una de las monedas de cambio más usuales, a lo largo de un eje de transformaciones que se polariza hacia la terminologización de ciertas palabras o bien hacia la trivialización de muchos términos especializados. Por lo tanto, encontramos una parte importante del léxico de la sostenibilidad que ya ha sido ampliamente divulgado en otros ámbitos especializados y no especializados, como podemos ver en los términos que hemos extraído de las publicaciones:

[...] emisiones toxicas, ahorro energético, consumo energético y consumo de energía, sobreconsumo de agua, materiales y energía, energías renovables, impacto ambiental, impacto medioambiental, reciclabilidad, sostenibilidad ambiental, política ambiental, generación de residuos, energía limpia, etiquetado ecológico, huella ecológica, agotamiento de recursos, residuos sólidos, cambio climático, acidificación atmosférica, compromiso ambiental.

Solo como apunte queremos destacar las numerosas polémicas que existen alrededor de la terminología del medio ambiente, ya que, como denuncian los colectivos ecologistas, a menudo se trata de una cierta perversión del lenguaje. Por ejemplo, el término energía limpia se utiliza para caracterizar la energía nuclear, lo cual es objeto de debate, puesto que ciertamente es una energía con bajas tasas de emisiones de gases de efecto invernadero pero genera desechos nucleares cuya eliminación no está resuelta. Es por esto que se habla de sucias energías limpias.

En cuanto a la terminología de la bioconstrucción, el mecanismo de derivación por prefijación es muy frecuente, especialmente con los prefijos eco- y bio-, propios de la terminología de conservación del medio ambiente, los cuales dan lugar a los términos: ecoaldea, ecotécnicas, ecoetiquetado, ecoetiquetas, ecodiseño, biohabitabilidad, bioconstrucción, bioconstructor y bioarquitectura. En el caso del último término, también encontramos la forma 
sintagmática arquitectura ecológica. La combinación de los dos prefijos la encontramos en las expresiones proyectos ecobioconstructivos y materiales ecobioconstructivos, que hacen referencia tanto a que no sean contaminantes como a que preserven el patrimonio natural e histórico del lugar. Destaca el sentido cognitivo de estos prefijos, ya que confieren un significado positivo a la palabra que acompañan.

La bioconstrucción genera nuevos términos con formantes cultos y mediante acronimia, esto es, términos formados por la combinación de segmentos de un sintagma desarrollado (Cabré, 1992: 165), como se puede ver en domobiótica, el cual da lugar a otros términos sintagmáticos y por acronimia: auditoría domobiótica, asesoramiento domobiótico, domopatías o patologías ambientales.

Además, la bioconstrucción ha dado lugar a la distinción entre casas ecológicas, bioconstrucciones y casas convencionales, estas últimas están definidas por contraposición a las anteriores, mediante un eufemismo para evitar decir contaminantes. De la misma manera, cuando se habla de materiales de construcción tradicionales, eufemísticamente, se hace también contraponiéndolos a los materiales sostenibles y reciclables. En el primer caso se habla de construcción convencional y, por contraste, en el segundo se trata de bioconstrucción. Como señala Salvador (2010: 151), el eufemismo maquilla una situación desagradable y la presenta bajo una nueva luz, en una especie de maniobra reparadora, que mitiga la crudeza de una sensación no deseada y reduce las amenazas por la imagen de los participantes, en el marco de la cortesía negativa.

Asimismo, encontramos numerosas antítesis en el discurso de la bioconstrucción, especialmente cuando se habla de energías, recursos y materiales. Por un lado, se utilizan los verbos minimizar, reducir o eliminar y, por otro lado, maximizar o aumentar. Lo mismo ocurre con los adjetivos energía renovable y no renovable, materiales peligrosos y no peligrosos y radioactivos, impacto minimo y elevado.

Queremos destacar dos de los adjetivos que mejor definen la bioconstrucción y la bioarquitectura, «respetuoso/a y sostenible: arquitectura saludable y respetuosa con el medio ambiente, construcción sostenible». También, leemos el título de un apartado "Viviendas pasivas y sostenibles», donde vivienda pasiva hace referencia a las viviendas que tienen un factor de sostenibilidad extraordinariamente alto. En este sentido, el término sostenibilidad se introduce en un artículo para hablar de un proyecto bioconstruido en el título: «Sostenibilidad vital en la Casa EX» y se explica de la forma siguiente: 
El sistema incorpora dispositivos de alta tecnología en los métodos de construcción y una predominante preocupación por la sostenibilidad en los procesos de obra y los materiales empleados, ofreciendo unos estándares ambientales y de terminaciones capaces de aunar un nivel de confort y bienestar contemporáneo con la recuperación del concepto de vida en el campo con todos sus atractivos. (9.b)

Aquí vemos cómo la sostenibilidad está contextualizada y relacionada semánticamente con procesos de obra, materiales, estándares ambientales, bienestar contemporáneo, concepto de vida en el campo.

Por otro lado, hay terminología específica de la bioconstrucción como por ejemplo: perfil ambiental del producto, ciclo de vida del producto, técnica Greb, mortero Greb, tejas de madera, geotermia, biogás, materiales biocompatibles, etc.

Finalmente, por tratarse de una disciplina con carácter conservacionista del medio ambiente, el color verde tiene un significado simbólico en algunas denominaciones referidas a conceptos constructivos. Así, encontramos la metodología VERDE, que sirve para la evaluación de la sostenibilidad en edificios y también el término sintagmático teja de madera verde, para referirse a un tipo de teja que no tiene tratamientos ni procesos añadidos.

\subsection{Las metáforas}

No son pocos los casos de metaforización en el discurso de la bioconstrucción, como ya hemos visto en la terminología. Aun así, la macrometáfora de la bioconstrucción es la consideración de un edificio como un ser vivo y, por esto, se le atribuyen características humanas y, así, se habla de la vida del edificio, la calidad de vida del inmueble, el edificio enfermo, edificio respetuoso con el entorno, salud y confort de los edificios. En la definición que propone el Decálogo de Bioconstrucción de la Asociación Española de Bioconstrucción, expone lo siguiente:

Tenemos la oportunidad de planear y construir edificios que respiren como nosotros, que podamos acariciar y saborear, que despidan un olor que nos haga crecer como seres, que la mirada se abra en su presencia, que se instalen en terrenos sanos y que cuando llegue el momento desaparezcan integrándose en el ciclo, como nosotros.

Se entiende por Bioconstrucción la forma de construir que favorece los procesos evolutivos de todo ser vivo así como la biodiversidad garantizando el equilibrio y la sustentabilidad de las generaciones futuras. (2.d) 
En este caso, la comparación «como nosotros» rige el primer párrafo, apareciendo en la primera frase para reforzar la metáfora «edificios que respiren» y vuelve en la última frase con la misma estructura y forma del inicio «como nosotros», con la finalidad de reforzar el sentido humanizador de los edificios bioconstruidos y con la función de cerrar textualmente el párrafo. El segundo y último párrafo se encargan de reformular el tema mediante una definición menos creativa y más ceñida al contexto científico.

Hemos visto que los edificios pueden estar enfermos, que queremos que respiren como nosotros, etc., y es tal la condición del edificio como un ser vivo que incluso puede estar cansado, lo cual lo hace todavía más humano, como se refleja en el siguiente título de apartado: «Cómo dar vida a un edificio cansado». Se emplea este adjetivo con el fin de explicar a continuación cómo un edificio industrial obsoleto y en mal estado se ha convertido en un centro cultural y social energéticamente eficiente, aunque conserve referencias visibles de su pasado como enclave comercial de primera línea.

El caso contrario viene de la consideración de los efectos de la electricidad no solo en los mecanismos eléctricos sino también en las personas. Veamos cómo describe un profesional el ambiente bioeléctrico de una casa cuando está muy alterado:

Este fenómeno electrostático ocurre también con asfalto y el hormigón (dieléctricos), que contribuyen a hacer estresantes e inhabitables nuestras ciudades, lo que justifica el título de mi libro Estrés de alta tensión, donde no me refiero solo a líneas de alta tensión eléctrica del cuerpo humano, que pasa fácilmente de los 100-500 mV sanos y biológicos hasta 15.000 y $20.000 \mathrm{mV}$ en un medio con contaminación electromagnética literalmente esto es alta tensión, y acabamos echando chispas! (4)

El mecanismo metafórico de «acabamos echando chispas!» que aparece al final del párrafo se establece partiendo de una característica relacionada con la electricidad y aplicándola a los seres humanos. Tiene la función de generar en la imaginación del lector el efecto que tiene en el cuerpo humano los elementos nocivos de la contaminación electromagnética de las viviendas actuales, una vez se ha citado el título del libro Estrés de alta tensión.

Por otro lado, en un proyecto de construcción de un parque infantil, la presencia de la metaforización se lleva a cabo con elementos cognitivamente externos a la construcción y se realiza tomando como elementos constitutivos de la metáfora los futuros usuarios de la instalación construida. Veámoslo: 
Y por último las características técnicas también fueron un argumento de peso, puesto que los suelos del parque infantil tienen que ser muy robustos para soportar bien el estrés mecánico de los piratas y las princesas que corretean por el mismo, los cochecitos que circulan y las torres de bloques que se derrumban. (9.c)

En el texto, la operación metafórica es «los niños son como los piratas» porque juegan a piratas o a ser piratas, y las niñas son como las princesas porque juegan a las princesas o a ser princesas. En esta generalización metafórica de los niños y de las niñas, estos dejan de serlo para pasar a ser piratas y princesas. Este mecanismo confiere al texto un toque divertido, y en sintonía con el objeto construido: «Un parque infantil de alegre amarillo», como dice el título del artículo.

\section{La ideología y la legitimación}

Como ya hemos apuntado anteriormente, la presencia de eufemismos léxicos es habitual en una disciplina que necesita presentarse menos contaminante de lo que ha sido y es todavía para abrirse camino entre la corriente actual de conservación del medio ambiente. Por ello, encontramos mecanismos discursivos de corrección política.

En primer lugar, veamos el título de un artículo, «Molienda ecosostenible» y el subtítulo, «Cómo proteger el medio ambiente y reducir los costes mediante el uso de aditivos para el cemento», donde molienda hace referencia al conjunto de materiales molidos que forman el cemento, material altamente contaminante en su elaboración. El adjetivo ecosostenible tiene un grado de subjetividad y de optimismo bastante alto, puesto que, como hemos visto antes en los significados de los prefijos, la presencia de los prefijos no implica la ausencia de contaminación, por lo que al adjetivo sostenible estos prefijos le confieren un significado excesivamente positivo, puesto que da a entender que medioambientalmente es inmejorable, cuando no es así. Luego, cuando se lee el artículo completo se entidende que supone una leve mejora medioambiental, pero está muy lejos de ser una solución.

El tema del cemento y del hormigón es relativamente polémico puesto que se trata de materiales contaminantes. Para tratar el tema de los materiales ecobioconstructivos, el autor de un artículo monográfico de la revista del Col-legi d'Aparelladors, Arquitectes Tècnics i Enginyers d'Edificació de Girona, destaca las ventajas y las desventajas que ha supuesto el cemento Pórtland, de la manera siguiente: 
El ciment Pòrtland, i sobretot el formigó armat va suposar un trencament amb els sistemes constructius anteriors, una revolució tecnològica que permeté nous llenguatges arquitectònics, noves formes d'organització social (ciutats dormitori, polígons d'habitatge...) i nous models econòmics. Tot i els seus grans avantatges, el seu ús massiu ha tingut greus conseqüències ambientals (alt cost energètic $i$ gran quantitat de residus i emissions) $i$ ha anat desplaçant els sistemes constructius anterios fins al punt que en molts casos s'ha traduït en una pèrdua dels coneixements de les tècniques tradicionals. Això, però, no vol ser un atac ferotge contra el formigó. És un toc d'atenció, ja que materials com la terra, la palla, la pedra o la fusta han quedat pràticament en desús, quan encara es podrien fer servir per a gran part dels usos dels que s'ha apoderat el formigó i l'acer. (8)

Se evita el conflicto con el lector y se busca la atenuación discursiva cuando se dice: «Això, però, no vol ser un atac ferotge contra el formigó. És un toc d'atenció». De esta manera, disminuye la crítica inicial, puesto que no quiere ser una ataque feroz contra el hormigón, sino un toque de atención. Evidentemente, en este tipo de revista profesional no se puede hacer un ataque desproporcionado a uno de los materiales de construcción que más ha contribuido a la profesión, como sería de esperar de un colectivo ecológico. No se debe olvidar que un colegio profesional es una organización de un grupo profesional que generalmente comparte una ideología profesional (Van Dijk, 2005b: 14). Aun así, las ideologías solo controlan el conocimiento a nivel general y global de grupo, es decir, el conocimiento común, y no necesariamente el conocimiento personal (Van Dijk, 2005a: 296).

Podemos ver la ideología de este discurso profesional en posturas claramente definidas como la que mostramos en el ejemplo siguiente, donde se aprovecha la introducción para exponer la amplia extensión del concepto y una evidente crítica social. Este ejemplo pertenece a la introducción de un artículo que lleva por título «Geobiología y bioconstrucción»:

He conocido personas que se acercan a la Geobiología desde la arquitectura, desde la agricultura en sus distintas versiones (biológica, clásica, permacultura...) desde la jardinería, desde diferentes ingenierías, desde el urbanismo, desde la biología, desde la geología, desde la hidrogeología y desde otros muchos ámbitos, como el de las personas que se interesan por las abejas, por los árboles, personas del mundo de la medicina. Es, sin duda, algo riquísimo. A veces trae sus complicaciones, pero es riquísimo. Porque todo este conjunto de personas tienen en común su absoluto amor por la tierra, lo entienda cada uno como lo entienda. Y todos tienen en común el deseo de expandir su consciencia y aprovechar sus capacidades no solo desde lo racional, sino a 
través del sentir, de los métodos biosensibles, para permitir que la intuición ocupe el lugar que le corresponde en su vida. Al fin y al cabo somos mucho más completos cuando utilizamos nuestros dos hemisferios cerebrales. [...] Ahora que está de moda la preocupación por el Medio Ambiente, de políticos poderosos que nunca se ocuparon de ello, prestemos especial atención al mensaje de miedo. [...] De ahí la importancia de estar atentos al mensaje que nos quieren hacer llegar. [...]

Cuando una persona decide construir su propia vivienda a veces con sus propias manos, con criterios de bioconstrucción, se está posicionando en una consciencia clara en relación con la naturaleza, con el medio, con las energías, con la vida y consigo mismo. (2.c)

Asimismo, también hay posturas menos amplias y procedentes de colegios profesionales, pero que marcan claramente el camino sociocognitivo medioambiental a seguir sin ningún tipo de duda:

La nostra feina, com a tècnics que creiem en un futur més sostenible de la construcció, consisteix a recuperar, investigar, assajar, comprovar aquests sistemes constructius i fer-ne difusió, amb la voluntat que, com s'ha fet en altres països, es puguin reconèixer com a materials habituals de les obres. (8)

En primer lugar, destaca el posesivo la nostra, en la nostra feina, que actúa de identificación del grupo y otorga un sentimiento de pertenencia al colectivo profesional (Van Dijk, 2005b: 14). Según el texto, la ideología profesional respecto a la conciencia medioambiental es común a todo el grupo profesional, puesto que los técnicos del ámbito de la construcción creen en un futuro más sostenible y por tanto son conscientes de ello y de la importancia que adquieren los nuevos sistemas, materiales, etc. La diferencia entre este fragmento y el anterior radica en el contexto de publicación, ya que mientras el primero se ubica dentro de la revista EcoHabitar, conocida por su carácter interdisciplinar, y con un objetivo claramente ecologista, donde diferentes profesionales buscan un futuro mejor para las personas y los lugares donde viven, el segundo se ha extraído de la revista de un colegio profesional de ingenieros técnicos en construcción. Así, podemos decir que el contexto determina que el discurso sea apropiado (Van Dijk, 2005b: 16).

Las creencias pierden su naturaleza ideológica en cuanto se convierten en parte del ideario social común (Van Dijk, 2005b: 11), pero en el discurso profesional de la bioconstrucción la creencia general de que se ha de conservar el medio ambiente se está concretando actualmente y se están elaborando los criterios que determinan lo que es o no es sostenible en ese ámbito. Se trata de 
un tema bastante polémico que enfrenta diferentes perspectivas, que implican intereses, disciplinas y profesionales de diverso orden.

Por otro lado, a menudo el mensaje es procedimental, ya que si no se hace lo que se recomienda, no se conseguirá una construcción y una vida más sostenible o no se cumplirá con la nueva normativa al respecto: «se tienen en cuenta criterios tan interesantes en relación a la salud, como el uso de los materiales más sanos y menos tóxicos posibles», «se tienen en cuenta criterios de los más respetuosos con el medioambiente, con el lugar» o «el objetivo a conseguir es un ambiente habitable y sano. Y en esto la bioconstrucción debe ser la apuesta de futuro, un futuro que debe ser ya presente».

Finalmente, hay que comentar las idas y venidas que se producen en el discurso de este tipo de disciplinas emergentes, cuando necesitan legitimarse pero consideran que tampoco se debe exagerar su alcance. Hay una presentación positiva de lo nuevo, pero a la vez hay un freno de lo que se dice, con efecto eufemístico de la información. En el último apartado del artículo citado anteriormente sobre la geobiología y la bioconstrucción, el autor dice: «la geobiología siempre nos puede permitir encontrar el lugar idóneo para ubicar una vivienda en una terreno dado», mientras que dos párrafos después añade: «Hemos de aclarar que la Geobiología no es una panacea llena de recetas que podemos aplicar en todo momento, o de cualquier manera». Al comienzo del siguiente párrafo indica: «El lugar es el que va a determinar hasta donde podemos llegar». Y, para acabar, al comienzo del último párrafo del artículo concluye: «Como todas las disciplinas, también la Geobiología puede tener sus límites ya que se trata de una práctica que está en constante evolución [...]».

\section{Conclusiones}

El discurso de la bioconstrucción se manifiesta en las revistas profesionales, de forma parcial o global, con el fin de introducir los nuevos parámetros medioambientales en esta disciplina, los cuales son definidos, explicados y argumentados como ideología común del grupo profesional. Esta introducción confiere al grupo profesional de la construcción y de la arquitectura, por un lado, la autoidentificación del grupo con los valores medioambientales y, por otro lado, la autoimagen, y la posterior imagen hacia el exterior, positiva por ser una disciplina acorde con los pensamientos conservacionistas actuales y por ser parte de las soluciones sostenibles.

La transmisión de la información y la concienciación del grupo profesional se lleva a cabo mediante un cierto grado de didacticidad, con el cual se definen 
las nuevas tecnologías y materiales, utilizando los términos medioambientales ya divulgados e introducidos ideológicamente en el sentido común con sentido positivo junto con la nueva terminología bioconstructiva. Para ello también se recurre a determinados mecanismos legitimadores de las nuevas ideas, empleando diversos mecanismos discursivos y léxicos como los eufemismos o las reformulaciones. En este sentido, los eufemismos y las reformulaciones hacen del discurso de la bioconstrucción un discurso compatible con el de la construcción sin generar enfrentamiento, sino integrando la nueva subdisciplina sostenible en la disciplina más tradicional y contaminante.

\section{Referencias bibliográficas}

Alcaraz, E. et al., (eds.) (2007): Las lenguas profesionales y académicas, Barcelona, Ariel.

BACH, C. (2001): «La reformulació en els textos d'especialitat» en BRUMME J. (ed.): La historia de los lenguajes iberorrománicos de especialidad. La divulgación de la ciencia, IULA-UPF-Vervuert-Iberoamericana: BarcelonaFráncfort-Madrid.

Cabré, M. T. (1992): La terminologia. La teoria. els mètodes, les aplicacions, Barcelona: Editorial Empúries.

- (1999): La terminología: representación y comunicación. Elementos para una teoría de base comunicativa y otros artículos, Barcelona: IULA-UPF.

GARCíA IzQuierdo, I. (2007): «Los géneros y las lenguas de especialidad» en Alcaraz, E. et al., (eds.), 119-126.

Louv, R. (2013): Volver a la naturaleza. El valor del mundo natural para recuperar la salud individual y comunitaria, Barcelona: RBA.

Montalt, V. (2005): Manual de traducció cientificotècnica, Vic: Eumo Editorial.

Montesinos, A. (2002): El discurs de la informàtica. Un estudi de les seqüències descriptives, València-Barcelona: IIFV-PAM.

Salvador, V. (2002): «Discurso periodístico y gestión social de los conocimientos», Anàlisi, n. ${ }^{\text {o }}$ 28: 107-120.

- (2009): «Pragmática de la definición en diversos géneros didácticos», en Siget, V. <http://cei.udc.es/uploads/archivos/files/\%5B11 \%5D\%20 Pragmatica\%20de\%20la\%20definicion.pdf $>$.

- (2010): «L'eufemisme, entre la retòrica i la política» en MARín, M. J. et al., (eds.) Discurs politic i identitats (trans)nacionals, Quaderns de Filologia, anejo . $^{\circ}$ 72, Universitat de València. 
- (2012): «Pràctiques discursives en l'àmbit sanitari: contextos, gèneres $\mathrm{i}$ estils», Llengua, Societat $i$ Comunicació, n. ${ }^{\circ}$ 10: 46-52.

- et al., (2010): «Divulgació i comunicació en la interacció clínica: Una aproximació des de la lingüística mèdica» en Edo N.; P. ORdóñez (eds.): El lenguaje de la ciencia y la tecnología, Castelló, UJ, 251-264. <http:// cei.udc.es/ uploads/archivos/files/[14]\%20Divulgació\%20i\%20comunicació $\% 20 \mathrm{cl} \% \mathrm{C} 3 \%$ ADnica $\% 20 . p d f>$.

SAlvador, V. et al. (2013): «La construcción de las profesiones sanitarias a través de las revistas especializadas», Discurso \& Sociedad, vol. 7 (1): 73-96. <http: www.dissoc.org>.

VAn Disk, T. (2005a): «Discurso, conocimiento e ideología. Reformulación de viejas cuestiones y propuesta de algunas soluciones nuevas», Cuadernos de Información y Comunicación, n. ${ }^{\circ}$ 10: 285-315.

- (2005b): «Ideología y análisis del discurso», Estudio, n. ${ }^{\circ}$ 29: 9-36.

Yus, F. (2007): «La comunicación en las lenguas de especialidad» en Alcaraz, E. et al. (eds.), 81-92.

\section{Anexo: Corpus}

\section{BIA/COAAT de Madrid. Bimestral}

«Nuevo planteamiento constructivo. Eficiencia y sostenibilidad», David Arias Arranz, 273 (verano 2012), 50-54.

2. EcoHabitar: Bioconstrucción, consumo ético, permacultura. Mensual

2.a «La técnica Greb: edificar con madera, paja y mortero de cal», Julio Tamata, 29, (primavera 2011), 22-25.

2.b «Tejados de madera: una solución tradicional para la bioconstrucción», Mike Harding, 25 (primavera 2010), 32-35.

2.c «Geobiología y bioconstrucción. 1a parte», Daniel Rubio, 17, (primavera 2008), 48-50.

2.d «Decálogo de Bioconstrucción», Asociación Española de Bioconstrucción, 13, (primavera 2007), 34-36.

3. La Punxa/COAAT de Girona. Trimestral «Materials ecobioconstructius sostenibles», Gabriel Barbeta Solà, 52, (2011), 20-27.

4. Inmueble. Revista del sector inmobiliario. Bimestral

«Bioconstrucción, vivienda sana y sostenible», Carlos M. Requejo, 28 (mayo-junio 2002), 89-95. 
5. Informes de la Construcción. Trimestral

«Iso 14006: Experiencias previas de estudios de arquitectura que han adoptado el estándar de ecodiseño UNE 150301-2003», G. Arana-Ladín; Cilleruelo, E.; Aldasoro, J. C., 527, vol. 64, julio-septiembre 2012, 319-330.

6. Revista técnica cemento y hormigón. Bimestral

«La sostenibilidad en la construcción: una visión práctica», Arturo Alarcón Barrio; Sergio Carrascón Ortiz, 948,( enero-febrero 2012), 66-75.

7. Arte y cemento, Revista de la construcción y su entorno. Quincenal

«Declaraciones ambientales de productos: materiales que definen a un edificio sostenible», Reportaje sostenibilidad, 7, (2012) 110-113.

8. TAG. Revista del Col-legi d'Aparelladors, Arquitectes Tècnics i Enginyers d'Edificació de Tarragona. Cuatrimestral

«Can Cargolí. Un espai per a la transformació ecosocial», Álvaro Pérez Otín, (1 ${ }^{\text {er }}$ quatrimestre 2013), 67, 20-21

9. Ecoconstrucción. Revista para los profesionales de la construcción, urbanismo, rehabilitación y diseño sostenible. Trimestral

9.a «Materiales para proyectos innovadores y sostenibles», (abril 2012), 32, 27.

9.b «Sostenibilidad vital en la Casa EX», (abril 2012), 32, 28.

9.c «Un parque infantil de alegre amarillo», (abril 2012), 32, 30-31.

9.d «Molienda ecosostenible», (mayo 2013), 35, 14-16.

9.e «Proyecto Aether: nueva gama de cementos con bajas emisiones», (mayo 2013), 35, 12-13. 